\title{
A Defect Detection Method for the Surface of Metal Materials Based on an Adaptive Ultrasound Pulse Excitation Device and Infrared Thermal Imaging Technology
}

\author{
Yibo Ai, ${ }^{1,2}$ Yingjie Zhang, ${ }^{1}$ Xingzhao Cao, ${ }^{1}$ and Weidong Zhang $\mathbb{D}^{1,2}$ \\ ${ }^{1}$ National Center for Materials Service Safety, University of Science and Technology Beijing, Beijing 100083, China \\ ${ }^{2}$ Southern Marine Science and Engineering Guangdong Laboratory, Zhuhai 519080, China \\ Correspondence should be addressed to Weidong Zhang; zwd@ustb.edu.cn
}

Received 13 May 2021; Revised 15 June 2021; Accepted 26 June 2021; Published 10 July 2021

Academic Editor: Chao Huang

Copyright (C) 2021 Yibo Ai et al. This is an open access article distributed under the Creative Commons Attribution License, which permits unrestricted use, distribution, and reproduction in any medium, provided the original work is properly cited.

Ultrasonic excitation has been widely used in the detection of microcracks on metal surfaces, but there are problems such as poor excitation effect of ultrasonic pulse, long time to reach the best excitation, and difficult to find microcracks. In this paper, an adaptive ultrasonic pulse excitation device and infrared thermal imaging technology have been combined, as well as their control method, to solve the problem. The adaptive ultrasonic pulse excitation device adds intelligent modules to realize automatic adjustment of detection parameters, which can quickly obtain reliable excitation; the multidegree-of-freedom base realizes the three-dimensional direction change of the ultrasonic gun to adapt to different excitation occasions. When the appropriate ultrasonic excitation makes microcracks in the resonance state, the microcracks can be frictionated, which produce heat rise with the temperature. Then, the microcrack defect can be detected by the infrared thermal instrument through the different surface temperatures with imaging recognition method. Our detection experiments of the titanium alloy plates and the aluminum alloy profiles of marine engineering show that the method can get reliable detection parameters in a short time and measure the crack length effectively. It can be used in many aspects such as crack detection in mechanical structures or complex equipment operating conditions and industrial production processes.

\section{Introduction}

Metal components are widely used in marine engineering, aerospace, vehicle engineering, and many other fields, and cracks and other defects are unavoidable in the process of production and operation. Therefore, the detection of cracks in metal parts of large-scale equipment has also put forward higher and higher requirements. Surface cracks are a common form of failure in metal components. If the cracks cannot be detected in time, the cracks will gradually expand, which will lead to the failure of components, which will lay a serious hidden danger for the safe operation of the equipment [1]. However, in engineering practice, microcracks on the metal surfaces are difficult to be directly observed by human eyes. Therefore, nondestructive testing methods such as ultrasound, radiation, penetration, infrared, and eddy current are often used for regular testing [2-6]. Among them, ultrasonic testing is suitable for various types of workpieces, which can detect surface cracks, internal cracks, and quantify cracks [7-11]. It is currently the most important method for crack detection and quantification [12].

Infrared thermal wave nondestructive detection is a specialized technology that uses the principle of infrared radiation to inspect and measure the surface of equipment or materials and other objects. It has the advantages of fast, large observation area, intuitive, accurate, noncontact, and other conventional detection technologies. It is suitable for field applications, online in-service testing, and so on [13-15]. Ultrasonic infrared thermal wave nondestructive detection technology uses the characteristics of ultrasound to transfer energy in the form of waves and inject it into the sample. If there are defects such as cracks, debonding, and 
tomography in the sample, friction, hysteresis, and thermoelastic effects will cause the injected energy to accumulate here and convert it into internal energy, which will increase the temperature. The changes in the temperature field will be captured by the infrared camera being inspected to identify defects. The commonly used ultrasonic infrared thermal imaging system consists of four parts: an ultrasonic excitation device, an infrared thermal imager, a computer microprocessor, and a measured object, as shown in Figure 1.

Scholars at home and abroad have conducted in-depth research on the use of ultrasonic infrared thermal wave technology to detect material surface cracks. Jia [16] built a nondestructive testing platform for rail tread cracks with infrared heat waves when inspecting rail foot cracks. According to the distribution of rail surface temperature information, the location of internal defects in the rail was determined. Jing et al., [17] used ultrasonic thermal wave imaging technology to detect cracks in locomotive knuckle parts. Experiments show that this technology is not sensitive to surface shape, rust, dust, and pollution and has special applications in the detection of defects such as cracks. Tang Schwarz et al. [18] simulated the heating phenomenon of austenitic stainless-steel weld cracks under ultrasonic excitation through the finite element analysis software Ansys and qualitatively analyzed the impact of ultrasonic excitation amplitude, frequency, direction, and weld reinforcement on the cracks. The authors in [19] proposed a new type of nondestructive testing method for composite structures of aluminum-carbon fiber composites. The interface between the three materials was characterized by ultrasonic and thermal imaging generated by an electromagnetic ultrasonic transducer. By detecting the artificial integration defects in the inner layer of carbon fiber composite materials, the quality of the interface bonding between the carbon fiber cloth and the thermoplastic layer is characterized.

The above analysis shows that infrared thermal wave as a new type of nondestructive testing technology has been widely favored by scholars, but there are still some problems to be solved when it is applied to the detection of metal cracks in engineering. The effect of ultrasonic infrared detection of cracks mainly depends on the three aspects of ultrasonic excitation energy and heat generation mechanism of crack action, parameters in the detection process, and signal processing of infrared heat maps. There is no scientific conclusion about the heat generation mechanism in the current research, and the mainstream friction heat generation mechanism still cannot perfectly explain all heat generated in the crack area [20-22]. The detection parameters include the parameters of the workpiece and the detection conditions of the ultrasonic infrared system. A large number of studies and experiments have shown that the detection parameters in the ultrasonic infrared nondestructive testing process have a significant impact on the detection effect, such as excitation frequency [23], excitation force [23], excitation time [24], and the difference between the horn and the tested part changes in the pretightening force [25] which will affect the transmission of ultrasound, affect the effect of ultrasound excitation, and cause large fluctuations in the test results. The signal processing of the infrared heat map is to process the infrared heat map obtained from the experiment to obtain intuitive and accurate crack information. In addition, the requirements for detection speed in metal crack defect detection and industrial production are increasingly high [26-29].

To improve the accuracy of crack defect detection, this paper starts researching from two aspects: optimal detection parameter selection and infrared heat map processing. An adaptive ultrasonic excitation device is designed to emit ultrasonic waves to the metal profiles so that its internal defects absorb and couple ultrasonic energy and produce local temperature imbalance areas. And the infrared imager is used to collect thermal images and crack information. In this way, it can diagnose the cracks in the production or use of metal profiles like titanium and aluminum of marine engineering equipment and solve the hidden safety problems caused by surface microcracks.

\section{Design of Ultrasonic Infrared Detection System}

The ultrasonic infrared detection system used in our research is shown in Figure 2, which mainly contains three major parts, which are an ultrasonic excitation system, an infrared thermal image detection system, and a data processing system.

2.1. Ultrasonic Excitation System. The ultrasonic excitation system is the adaptive ultrasonic excitation device proposed in this paper. At present, there are two main types of ultrasonic excitation devices such as one is the vertical ultrasound excitation device and the other is the handheld ultrasound excitation device. The vertical ultrasonic excitation device has a large structure, high power, stable and reliable excitation effect, and high efficiency and is easy to operate automatically, but it costs a lot. The handheld ultrasonic excitation device is small in structure, easy to move, can adapt to the ultrasonic excitation of workpieces in multiple locations, and has low testing cost. However, it has small power, poor excitation effect, low detection efficiency, and limited application. Therefore, it is of great practical value to research and design an ultrasonic excitation device with small structure, easy operation, and good excitation effect to achieve the best excitation effect. The adaptive ultrasonic excitation device proposed in this study can overcome the abovementioned problems, and the multidegree-of-freedom base of the ultrasonic gun enables the device to adapt to different operating environments and meet the needs of actual use.

This paper designs an adaptive ultrasonic pulse excitation device, and its structure and toolchain are shown in Figure 3. The device includes an ultrasonic controller and a multidegree-of-freedom base of the ultrasonic gun. The ultrasonic controller includes an ultrasonic generator and an ultrasonic transducer. Among them, the ultrasonic generator adopts the stepless frequency modulation ultrasonic generator, the frequency range is $13 \mathrm{kHz} \sim 75 \mathrm{kHz}$, which can be modulated by $0.1 \mathrm{kHz}$, and the ultrasonic transducer 
frequency is $20 \mathrm{kHz}$. The ultrasonic generator is connected to the ultrasonic transducer through a line, and the working parameters of the ultrasonic generator are adjusted through adaptive control to quickly obtain better ultrasonic excitation effects. The multidegree-of-freedom base of the ultrasonic gun can adapt to different operating environments so that the device can meet the actual needs. The device adopts the principle of information feedback, which can ensure that reliable experimental parameters (excitation frequency, excitation power, excitation time, compression force between the horn and the tested time, incentive position, and other parameters) are obtained in a short time.

The ultrasonic controller of the adaptive pulse excitation device includes the control panel and the internal functional modules of the ultrasonic controller. The ultrasonic controller control panel includes the power switch, the auto/ manual mode switch button, and the ultrasonic controller. The control panel includes pulse excitation frequency adjustment knob and its display screen, ultrasonic pulse excitation power adjustment knob and its display screen, ultrasonic pulse excitation time adjustment knob and its display screen, and the compression force adjustment knob between the ultrasonic gun horn and the test piece and its display screen; the internal functional module of the ultrasonic controller includes four major modules, which are parameter manual control module, parameter automatic control module, parameter feedback module, and data access module. The ultrasonic controller powers the circuit board through the power switch, selects the auto/manual mode, adjusts the experimental parameters according to the corresponding mode, and starts the work of each module; among them, each parameter adjustment knob and display screen is connected to the parameter manual control module.

The multidegree-of-freedom base of the ultrasonic gun of the adaptive pulse excitation device includes moving wheels, chassis, plane rotating frame and its driving mechanism, height rising frame and its driving mechanism, angle tilt frame and its auxiliary mechanism; ultrasonic gun card tool and base control panel are mainly used to fix the direction and position of the ultrasonic gun. Under the multidegree-of-freedom base chassis of the ultrasonic gun, there are moving wheels. The front surface of the chassis has a base control panel. The base control panel is connected to three driving mechanisms: plane rotation, height rise, and angle elevation. The plane rotating frame and its driving mechanism are placed on the chassis. The rotating frame and its driving mechanism are equipped with a height raising frame and its driving mechanism. The upper part of the height raising frame and its driving mechanism is the angle pitch frame and its auxiliary mechanism and ultrasonic gun fixture; the ultrasonic gun fixture includes a servo motor, lead screw, and threaded clamping cylinder. The servo motor can drive the lead screw to expand and contract the threaded clamping cylinder that is screwed to the screw. A force sensor is placed in front of the ultrasonic gun to transmit the pressure measurement value to the ultrasonic controller. The force sensor adopts the S-type load cell MIK-LCS1, with a range of $0-200 \mathrm{~kg}$ and an accuracy of $0.03 \%$ FS.
2.2. Infrared Thermal Image Detection System. The infrared thermal image detection system mainly includes an infrared thermal imager, its auxiliary equipment, and data processing system.

The infrared thermal imager uses FLUKE Ti10 handheld thermal imager with a resolution of $640 \times 480,1.3$ million pixels, and a thermal sensitivity (NETD) of $200 \mathrm{mK}$ at $30^{\circ} \mathrm{C}$. After ultrasonic excitation, the surface temperature of the surface crack will be higher than that of other locations because of the friction. The temperature change at the crack defect is greater, which leads to the uneven distribution of the temperature field on the surface of the alloy profile. The infrared imager is used for detection, and the temperature of different areas can be displayed intuitively.

The data processing system analyzes, processes, and evaluates the collected image data. Users can interact with other systems through the data processing system and can also perform image processing, data management, and other functions through related software operations. The software includes functions such as image reading and accessing, heat image grayscale processing, crack contour extraction, and crack length measurement. The software interface is shown in Figure 4. The image processing mainly includes operations such as image background difference, image segmentation, feature extraction, defect recognition, and defect reconstruction on the collected infrared heat map. The processing flow is shown in Figure 5.

\section{Control Method of Adaptive Ultrasonic Pulse Excitation Device}

This paper proposes an adaptive ultrasonic pulse excitation device control method. For the internal function modules of ultrasonic control, including parameter manual control module, parameter automatic control module, parameter feedback module, and data storage module, there are two control methods: manual control mode and automatic control mode.

3.1. Manual Control Mode of Adaptive Ultrasonic Pulse Excitation Device. Manual control mode includes the ultrasonic controller parameter manual control module, parameter feedback module, and data access module. The frequency adjustment knob, power adjustment knob, time adjustment knob, and compression force adjustment knob between the horn and the test piece on the ultrasonic controller panel are manually adjusted, and the module reads the set parameters and then passes through the module; each submodule output (including frequency control submodule, power control submodule, time control submodule, and compression force submodule between the horn and the tested sample) would be transferred as highfrequency alternating current and signals that meet the set parameters, and it is transmitted to the ultrasonic gun and its multidegree-of-freedom base so that the ultrasonic gun can emit ultrasonic waves with set frequency, power, and excitation time. At the same time, the parameter feedback module (as shown in Figure 6) also reads the set parameters 


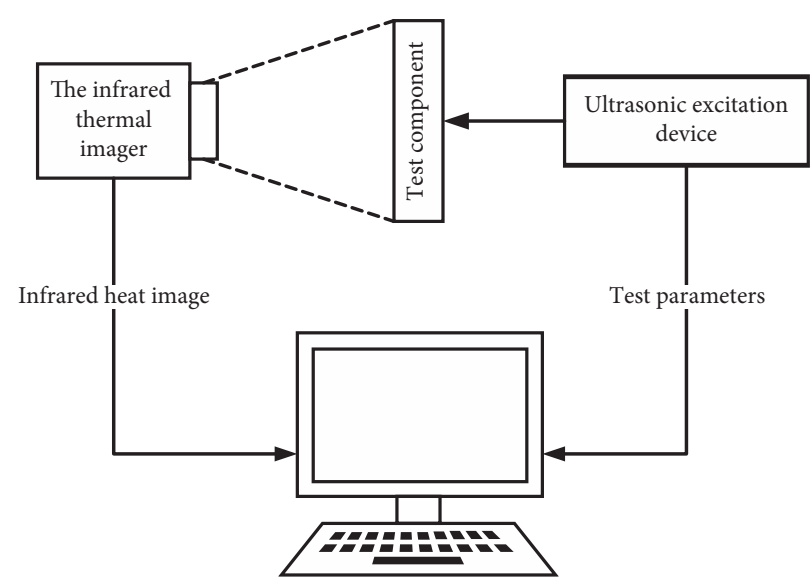

FIgURE 1: Composition of the ultrasound excitation infrared thermal imaging system.
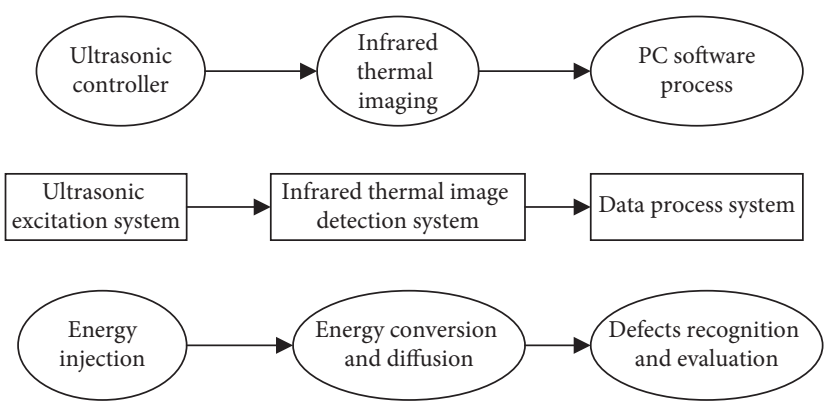

FIGURE 2: Relationship diagram of ultrasonic and infrared nondestructive testing equipment.

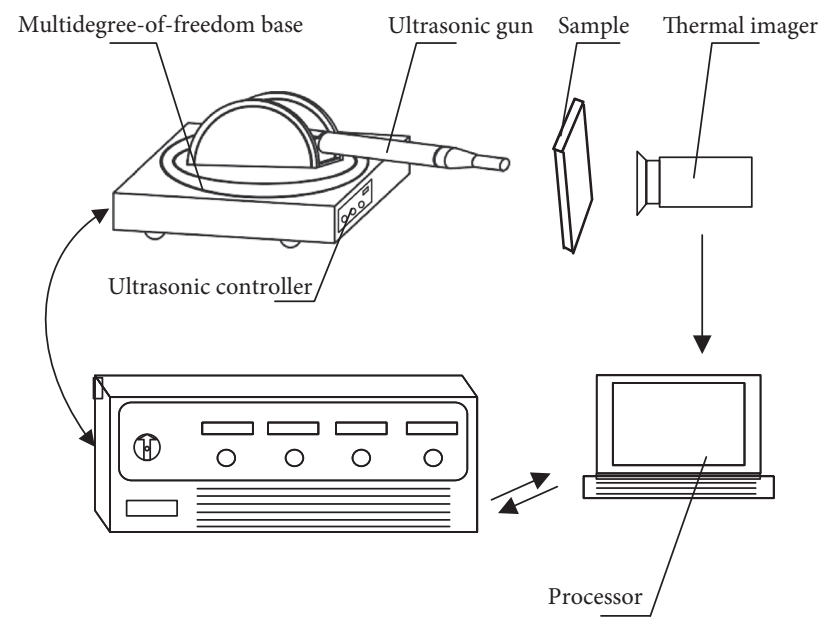

Figure 3: Schematic diagram of the adaptive ultrasonic pulse excitation device and its working chain.

and receives the processed excitation data obtained by the infrared thermal imager from the microprocessor. After comprehensive analysis and evaluation of the data, the data are stored in the data access module.

The data access module in Figure 7 mainly consists of two parts: the corresponding experimental conditions, including the relevant parameters of ultrasonic excitation, and the data information for subsequent infrared image processing.

\subsection{Automatic Control Mode of Adaptive Ultrasonic Pulse} Excitation Device. The automatic control mode includes parameter automatic control module, parameter manual control module, parameter feedback module, and data storage module. The flow chart of automatic control mode is shown in Figure 8. follows:

The working steps of automatic control mode are as 


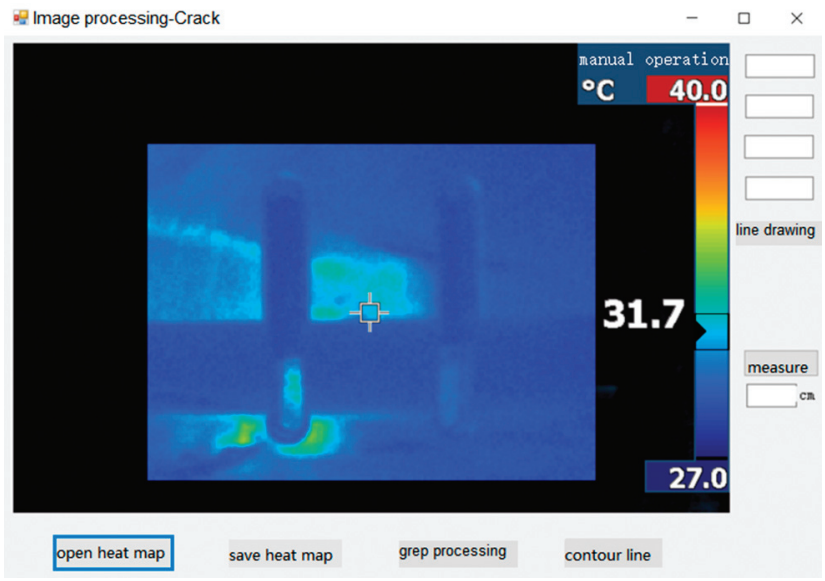

Figure 4: Data processing software interface.

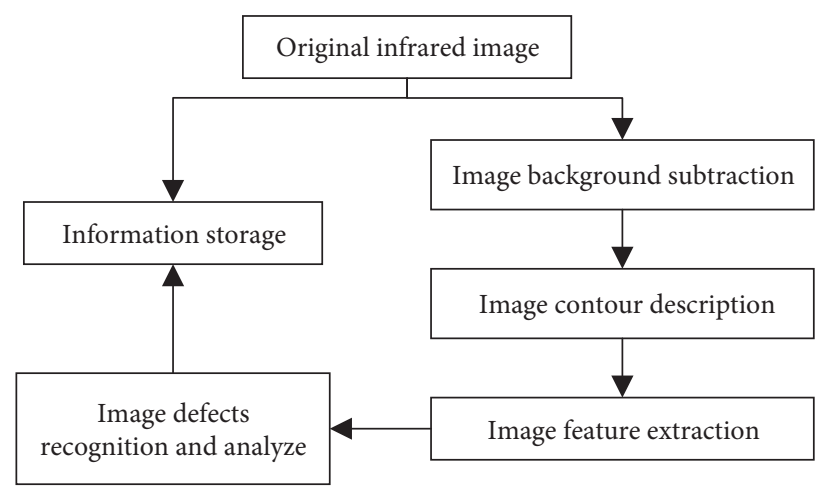

FIgURE 5: Thermal image data processing flow chart.

(1) The automatic control mode is selected under the ultrasonic controller panel, and the parameters feedback module reads a set of existing optimal working parameters from the data access module and transmits them as the initial working parameters to the parameters automatic module.

(2) After the data conversion of the parameter automatic control module, the signal is transferred to the circuit conversion module of the parameter manual control module, adjusted by the submodules in the parameter manual control module (including frequency control submodule, power control submodule, time control submodule, and compression force submodule between the horn and the tested sample). The output high-frequency alternating current and signals that meet the set parameters will be transmitted to the ultrasonic gun and its multidegree-of-freedom base.

(3) The parameter feedback module receives the excitation data and judges whether the range of the best excitation effect has been reached after analysis and evaluation. If the range of the best excitation effect is reached, further excitation is stopped and the data are written into the data storage. If the range of the best excitation effect is not reached, the modified experimental parameters will be given and passed to the parameter automatic control module.

(4) Repeat steps (1) to (3) until the best excitation effect is achieved; when the requirements are met, the parameters are stored in the data access module and stop working.

Combining manual control mode and automatic control mode to realize ultrasonic infrared nondestructive testing is an important innovation of this article. It can effectively solve the shortcomings of the traditional ultrasonic pulse excitation device with poor excitation effect and long time to achieve the best excitation effect. At the same time, there are many newly designed ultrasonic guns. The degree-of-freedom base can meet the needs of use in complex environments.

\section{Experiments and Results Discussion}

To verify the effectiveness of the adaptive ultrasonic excitation device and its control method proposed in this paper, a crack detection experiment on the alloy was carried out. The proposed method is a nondestructive crack detection method for metal surfaces, and there is no strictly special size of the sample and crack size. The experiment uses the ultrasonic infrared nondestructive testing system in Figure 2. 


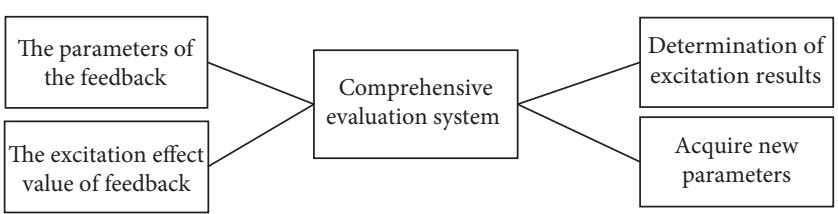

FIGURE 6: Schematic diagram of the working principle of parameter feedback module.

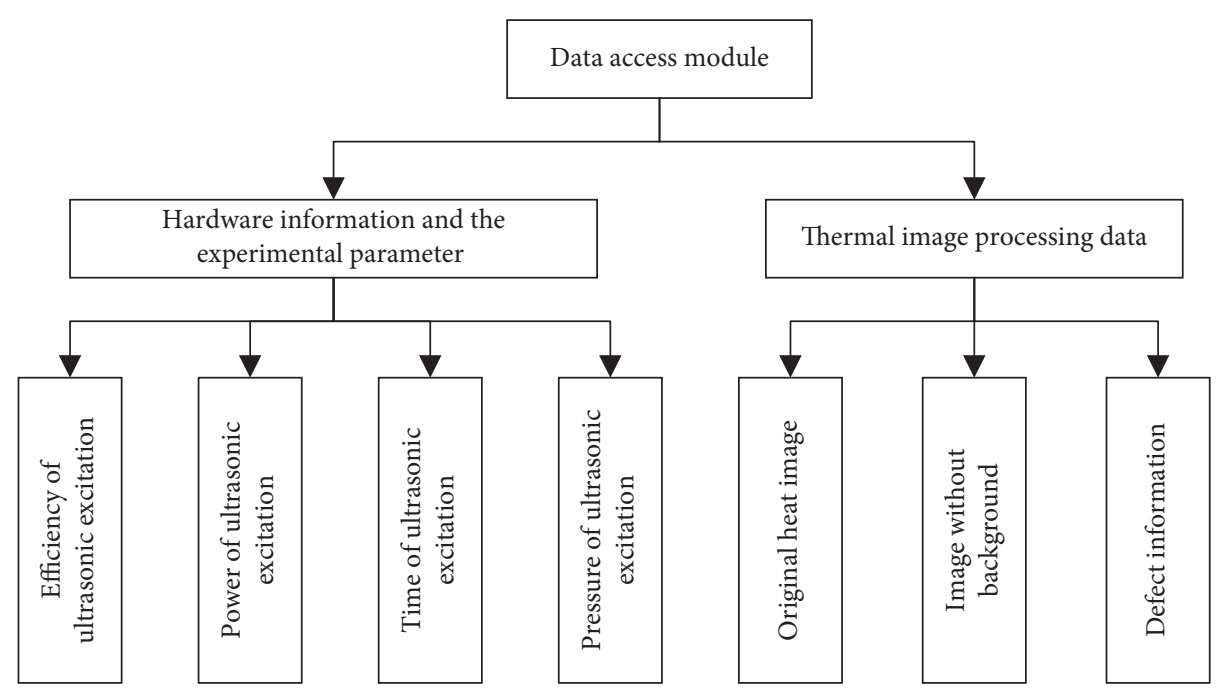

FIgURE 7: Schematic diagram of data access module.

The experiment is to detect the crack of U-groove specimen after the fatigue test. A sample is shown in Figure 9. The excitation gun is contacting the sample on one edge of the sample because the edge is flat and is easy to tough the gun. And for the placement, it is easy to detect the cracks by the infrared thermal imager at a positive perspective.

The ultrasonic excitation system is the active energy source for the crack detection experiment of metal material profile. The multidegree-of-freedom base of the ultrasonic gun is moved, and the excitation parameters are tested accordingly. The parameter feedback module reads a set of existing optimal working parameters from the data access module as the initial working parameters and passes them to the parameter automatic control module. Then, the data of the parameter automatic control module will be conversed and be transferred to the circuit conversion module of the parameter manual control module. After the control of each submodule in the parameter manual control module, it outputs high-frequency alternating current and signals that meet the set parameters and transmits them to the ultrasonic gun and multidegree freedom base. Then, the parameter feedback module receives, analyzes, and evaluates the excitation data. It is judged whether the range of the best excitation effect has been reached. The excitation will be stopped when the data access module reaches the range of the best excitation effect. The modified experimental parameters will be given and passed to the parameter automatic control module. After reaching the requirement, the parameter will be written into the data access module and stop working.
The multidegree-of-freedom base of the ultrasonic gun is moved to a suitable position and fixed. The orientation of the three rotating frames is adjusted according to the actual situation, the pressure control signal from the ultrasonic controller is received to control the rotation angular displacement of the servo motor, and then the threaded cartridge is pushed by the screw to move forward to obtain the set pressing force. The pressing force can be measured by a force sensor placed in front of the ultrasonic gun fixture. When the pressing force is not suitable, the force sensor transmits the measured value to the ultrasonic controller for observation and adjustment.

Parts of the images collected by the experiment are shown in Figure 10. The grayscale images of the excitation heat maps and the measured crack length after processing by the data processing system are shown in Figure 11. The crack can be observed more clearly, and its length can be measured through the following equation:

$$
l=\sqrt{\left(x_{1}-x_{2}\right)^{2}+\left(y_{1}-y_{1}\right)^{2}} .
$$

Finally, a database is established to record the experimental data, including the original experimental conditions (relevant parameters of ultrasonic excitation and so on), the original heat maps, and the final thermal images defect identification information.

It can be seen that the proposed adaptive ultrasonic infrared crack defect detection system can identify defects in real time. The heat produced by the cracks' friction after receiving the ultrasonic excitation can be captured by the infrared thermal imager. A software has been developed to 


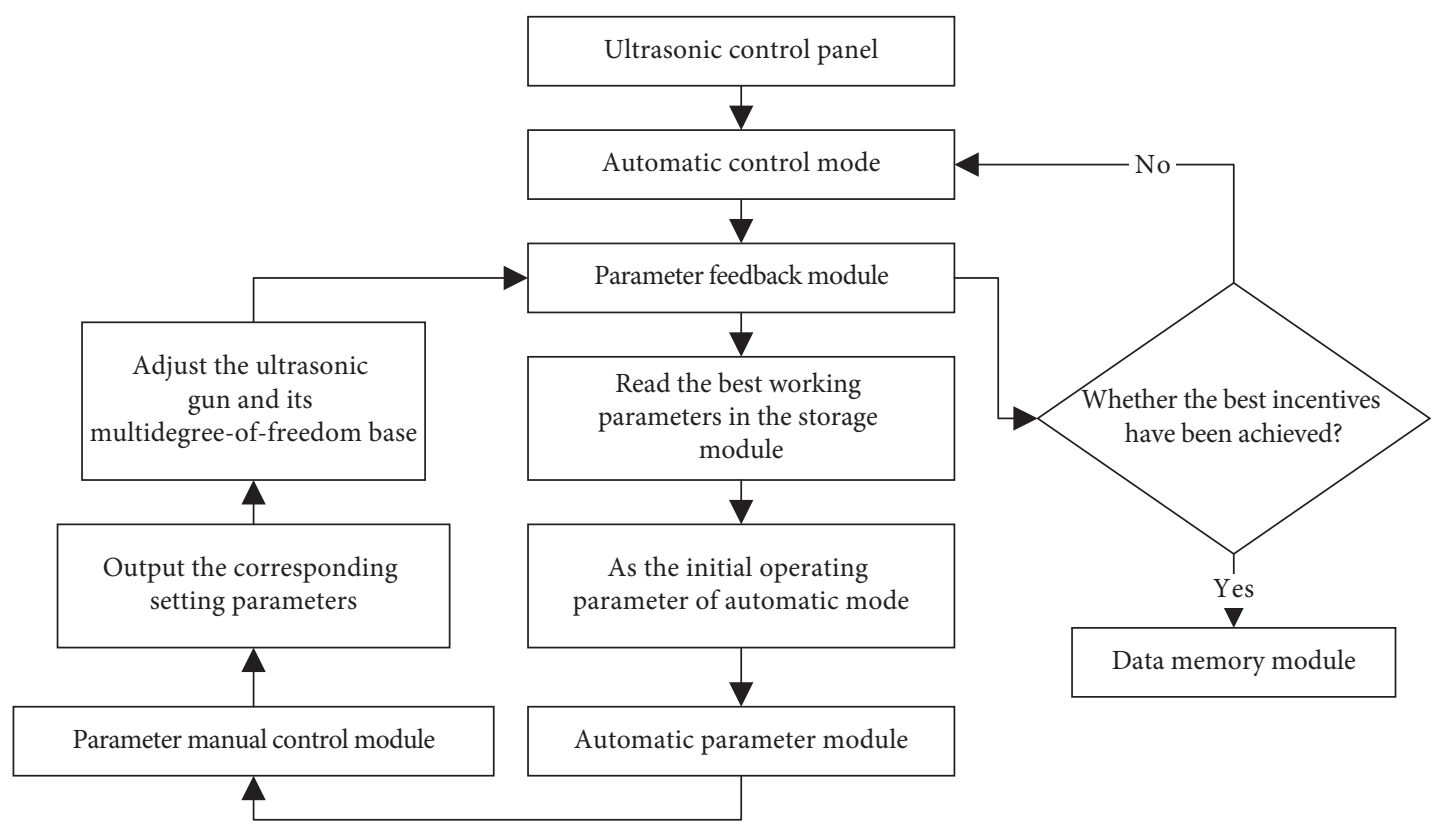

Figure 8: Flow chart of automatic control mode.

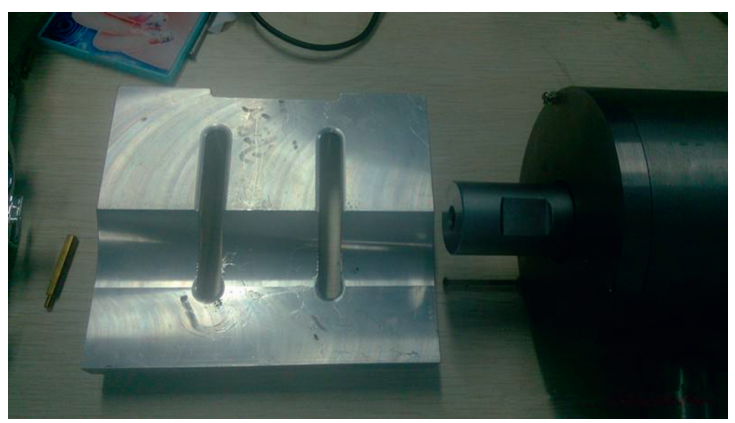

Figure 9: The sample.

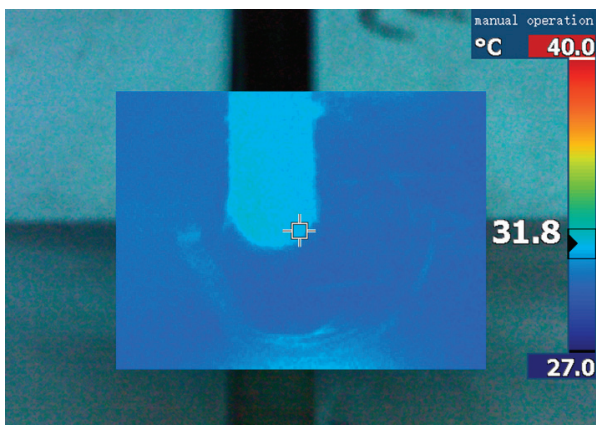

(a)

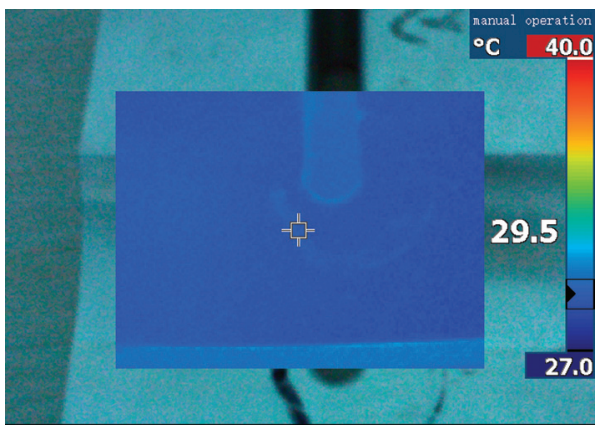

(b)

FIgURE 10: Part of experimental acquisition images. 


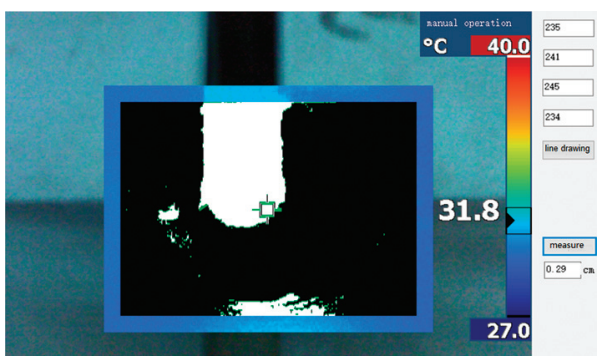

(a)

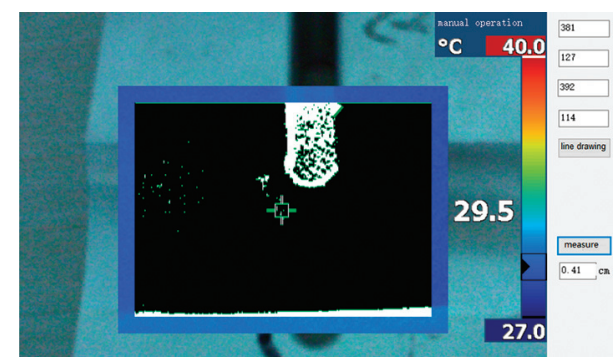

(b)

FIgURE 11: The processed images and the crack length.

recognize the defect from the thermal map and provide image process operation choice. Therefore, the defect can be detected, and its size can be actually calculated easily.

\section{Conclusions}

This paper designs an adaptive ultrasonic excitation device and its control method. The ultrasonic controller based on the original parameter manual control module adds a parameter feedback module, parameter automatic control module, and data access module. The main function of the parameter feedback module is to collect the current equipment working parameters and evaluate and judge the working parameters for the parameters setting of the next step when the equipment is running; the parameter automatic control module is corresponded to the parameter manual control module. With the assistance of the parameter feedback module and data access module, it can automatically correct the working parameters of the equipment and quickly obtain the actual best optimal working parameters; the data access module is mainly to output the best optimal working parameter. It can store the optimal working parameter and write to the parameter feedback module.

Compared with the existing ultrasonic excitation device, this device has the advantages of small device structure, convenient operation, and quicker obtaining the best excitation effect. At the same time, the multidegree-of-freedom base of the ultrasonic gun can adapt to different operating environments and meet the needs of actual use. Our quantitative detection experiments on the surface cracks of metal sheets prove that this technology can detect contact interface defects effectively such as fatigue cracks in composite materials. It can well solve the problem of identifying defects in parts of the metal profile and plates in service environments. And it can also be applied widely in industrial production.

\section{Data Availability}

The relevant data in this article were obtained by the author's personal investigation and research and can be made available upon request.

\section{Additional Points}

Featured Application. This paper provides a nondestructive detection method for microcracks on the surface of metal materials. Ultrasound excitation will be applied to the surface of the metal material, and the infrared thermal instrument will be used to detect the defect through the improved adaptive infrared imaging recognition method.

\section{Conflicts of Interest}

The authors declare that they have no conflicts of interest.

\section{Authors' Contributions}

Yibo Ai and Weidong Zhang conceived and designed the study. Yibo Ai and Xingzhao Cao designed the device and developed the software. Yingjie Zhang gathered the data. Yibo Ai and Yingjie Zhang wrote the paper. All authors read and approved the manuscript. Yibo Ai and Yingjie Zhang contributed equally to this work.

\section{Acknowledgments}

This work was supported by the Innovation Group Project of Southern Marine Science and Engineering Guangdong Laboratory (Zhuhai) (No. 311021013), the Fundamental Research Funds for the Central Universities of China (Nos. FRF-GF-20-24B and FRF-MP-19-014), and the 111 Project (No. B12012).

\section{References}

[1] D. Wang, Fracture Mechanics, Harbin Institute of Technology Press, Harbin, China, 1989.

[2] K. C. Kim, J. Y. Kwon, and N. W. Kang, "A novel forcedresonance microwave method to detect surface cracks in metal," Ieice Electronics Express, vol. 13, no. 17, Article ID 20160715, 2016.

[3] Q. Tang, C. Bu, Y. Liu et al., "Computer simulation of metal surface micro-crack inspection using pulsed laser thermography," International Journal of Multimedia \& Ubiquitous Engineering, vol. 3, no. 11, pp. 249-256, 2016.

[4] S.-H. Yang, K.-B. Kim, and J.-S. Kang, "Detection of surface crack in film-coated metals using an open-ended coaxial line sensor and dual microwave frequencies," NDT \& E International, vol. 54, no. 54, pp. 91-95, 2013.

[5] C. Xu, X. Gong, W. Zhang, and G. Chen, "An investigation on eddy current pulsed thermography to detect surface cracks on the tungsten carbide matrix of polycrystalline diamond compact bit," Applied Sciences, vol. 7, no. 4, p. 429, 2017. 
[6] W. Zhu, Z. Liu, D. Jiao et al., "Eddy current thermography with adaptive carrier algorithm for non-destructive testing of debonding defects in thermal barrier coatings," Journal of Nondestructive Evaluation, vol. 3137 pages, 2018.

[7] X. Kou, C. Pei, and Z. Chen, "Fully noncontact inspection of closed surface crack with nonlinear laser ultrasonic testing method," Ultrasonics, vol. 114, Article ID 106426, 2021.

[8] L. Feng and X. Qian, "Enhanced sizing for surface cracks in welded tubular joints using ultrasonic phased array and image processing," NDT \& E International, vol. 116, Article ID 102334, 2020.

[9] Z. A. Wei, A. Sq, L. A. Li et al., "An quantitative inspection method for internal defects based on laser ultrasonic technology," Optik, vol. 216, Article ID 164873, 2020.

[10] A. Messager, A. Junet, T. Palin-Luc et al., "In situ synchrotron ultrasonic fatigue testing device for 3D characterisation of internal crack initiation and growth," Fatigue \& Fracture of Engineering Materials \& Structures, vol. 3, no. 43, pp. 558567, 2020.

[11] Y. Zhu, F. Li, and W. Bao, "Fatigue crack detection under the vibration condition based on ultrasonic guided waves," Structural Health Monitoring, vol. 3, no. 20, pp. 931-941, 2021.

[12] Z. X. Zhou, "Overview of NDT methods for mechanical cracks," Journal of Mechanical \& Electrical Engineering, vol. 10, no. 34, 2017.

[13] G. Dua, V. Arora, and R. Mulaveesala, "Defect detection capabilities of pulse compression based infrared non-destructive testing and evaluation," IEEE Sensors Journal, vol. 21, no. 6, pp. 7940-7947, 2020.

[14] Z. Qu, P. Jiang, and W. Zhang, "Development and application of infrared thermography non-destructive testing techniques," Sensors, vol. 14, no. 20, p. 3851, 2020.

[15] F. Flora, M. Boccaccio, G. Fierro et al., "Non-destructive thermography-based system for damage localisation and characterisation during induction welding of thermoplastic composites," Thermosense: Thermal Infrared Applications XLII, vol. 11409, Article ID 114090I, 2020.

[16] W. Jia, "Experiment into nondestructive testing of rail foot cracks using infrared thermal waves," Infrared Technology, vol. 2, no. 42, pp. 163-167, 2020.

[17] F. Jing, L. Peng, H. Jiang, L. Chen, and W. Yibing, "Crack detection of locomotive hook tongue based on ultrasonic thermography," Infrared Technology, vol. 2, no. 42, pp. 158$162,2020$.

[18] M. Schwarz, M. Schwarz, S. Herter et al., "Nondestructive testing of a complex aluminium-CFRP hybrid structure with EMAT and thermography," Journal of Nondestructive Evaluation, vol. 38, no. 1, 35 pages, 2019.

[19] S. D. Holland, L. Koester, J. Vaddi et al., "VibroSim: a hybrid computational/empirical model of vibrothermography nondestructive evaluation," Review of Progress in Quantitative Nondestructive Evaluation: Incorporating the EuropeanAmerican Workshop on Reliability of NDE, vol. 1706, pp. 249-276, Article ID 100008, 2016.

[20] J. Vaddi, S. D. Holland, and R. Reusser, "Transducer degradation and high amplitude behavior of broadband piezoelectric stack transducer for vibrothermography," Review of Progress in Quantitative Nondestructive Evaluation, vol. 31, no. 1430, pp. 552-558, 2012.

[21] Lesthaeghe, "Evaluation of some parameters influencing vibrothermographic crack heating," Dissertations \& TheseGradworks, Iowa State University, Ames, IA, USA, 2015.
[22] F. Ma and X. Guo, "Modeling and analysisi of vibrotherography for the detection of microcracks," Nondestructive Testing, vol. 9, no. 37, pp. 6-10, 2015.

[23] C. Zhang, A. Song, F. Fuzhou et al., "Study on optimization methods of ultrasonic infrared thermography detection conditions," Infrared and Laser Engineering, vol. 2, no. 45, pp. 77-84, 2016.

[24] X. Han and Y. Song, "Study the effect of engagement force of ultrasound transducer on crack detectability in sonic IR imaging," AIP Conference Proceedings, vol. 1, no. 1511, pp. 532-538, 2013.

[25] F. Fuzhou, C. Zhang, Q. Min et al., "Heating characteristics of metal plate crack in sonic IR imaging," Infrared and Laser Engineering, vol. 5, no. 44, pp. 1456-1461, 2015.

[26] J. P. Yun, W. C. Shin, G. Koo, M. S. Kim, C. Lee, and S. J. Lee, "Automated defect inspection system for metal surfaces based on deep learning and data augmentation," Journal of Manufacturing Systems, vol. 55, pp. 317-324, 2020.

[27] H. Gao, X. Qin, R. J. D. Barroso et al., "Collaborative learningbased industrial IoT API recommendation for software-defined devices: the implicit knowledge discovery perspective," IEEE Transactions on Emerging Topics in Computational Intelligence, vol. 99, pp. 1-11, 2020.

[28] H. Gao, W. Huang, and Y. Duan, "The cloud-edge-based dynamic reconfiguration to service workflow for mobile ecommerce environments," ACM Transactions on Internet Technology, vol. 21, no. 6, 2021.

[29] L. Kuang, T. Gong, S. OuYang, H. Gao, and S. Deng, "Offloading decision methods for multiple users with structured tasks in edge computing for smart cities," Future Generation Computer Systems, vol. 105, pp. 717-729, 2020. 